Research Group: Macroeconomics

décembre 2009

\title{
Une estimation de la cible implicite d'inflation dans la zone Euro
}

\author{
PATRICK FÈVE, JULIEN MATHERON \\ et Jean-Guillaume SAHUC
}




\title{
Une estimation de la cible implicite d'inflation dans la zone euro ${ }^{1}$
}

\author{
Patrick Fève, \\ TSE (GREMAQ-IDEI, et IUF) et Banque de France
}

Julien Matheron,

Banque de France et SDFi, Université de Paris-Dauphine

Jean-Guillaume Sahuc ${ }^{2}$

Banque de France et Audencia, Ecole de Management

\footnotetext{
${ }^{1}$ Nous tenons à remercier $P$. Casati (Editeur) ainsi qu'un rapporteur anonyme de la revue pour leurs commentaires et suggestions. Les vues exprimées dans cet article sont celles des auteurs et ne reflètent pas nécessairement celles de la Banque de France.

${ }^{2}$ Banque de France, DGEI-DEMS-SEPS UA 46-2403, 31 rue Croix des Petits Champs, 75049 Paris cedex 01. Email : jean-guillaume.sahuc@banquefrance.fr
} 


\title{
Résumé
}

Le taux d'inflation dans l'ensemble des pays qui constituent la zone euro a fortement diminué au cours des années 1980. Durant cette période, le taux de chômage a significativement augmenté et l'activité économique a notablement ralenti dans la zone. Les variations de la cible implicite d'inflation des banques centrales, assimilée aux mouvements de basse fréquence de l'inflation, sont potentiellement un facteur d'explication de ces évolutions.

Afin d'éclairer cette question, cette étude propose d'estimer les évolutions dynamiques de la cible implicite d'inflation dans la zone euro au cours de la période 1970(1)-2004(4) à l'aide d'une petite maquette macro-économétrique. Cette cible implicite d'inflation, inobservée de l'économiste, est identifiée à l'aide d'un ensemble minimal de restrictions théoriques: (i) la cible implicite d'inflation suit un processus non stationnaire (ii) l'inflation est un phénomène exclusivement monétaire à long terme et (iii) les variations de la cible implicite d'inflation n'ont pas d'effet réel à long terme.

Le modèle est estimé de façon à reproduire le taux de croissance du PIB, la variation de l'inflation et le taux d'intérêt réel ex post. Les résultats principaux qui se dégagent de l'analyse empirique sont les suivants: (i) les chocs sur la cible implicite d'inflation expliquent l'essentiel des fluctuations des variables nominales, même à court terme; (ii) leurs effets réels à court terme transitent par l'inertie du taux d'intérêt nominal et la forte hausse du taux d'intérêt réel qui en découle ; (iii) en dépit de cela, ces chocs n'affectent que modestement la dynamique du PIB.

Mots-clés : Cible implicite d'inflation, modélisation macro-économétrique, zone euro Codes JEL : $\quad$ E31, E32, E52

\begin{abstract}
Euro area countries as a whole have experienced a marked downward trend over the 1980s. Over this period, the unemployment rate has increased and economic activity has been sluggish. Changes in the implicit inflation target, viewed as low frequency movements of inflation, might possibly explain these developments. To highlight this issue, the present study estimates the dynamics of the implicit inflation target in the euro zone over the period 1970Q1-2004Q4. Based on a small macroeconometric model, the implicit target, not known by the econometrician, is identified through a minimal set of theoretical restrictions: (i) the inflation target is a non stationary process, (ii) inflation is a monetary phenomenon in the long-run, and (iii) changes in the implicit target have no long-run effects whatsoever on real variables.

The model is estimated so as to match output growth, changes in inflation and the ex post real interest rate. Our main results are: (i) inflation target shocks account for the bulk of nominal fluctuations; (ii) due to monetary policy inertia and nominal stickiness, changes in the target generate large swings in the real interest rate translating into substantial short-run effects on real variables; (ii) in spite of this inflation target shocks moderately impact on output dynamics.
\end{abstract}

Keywords: Implicit inflation target, Macroeconometric modelling, Euro area JEL Codes: $\quad$ E31, E32, E52 


\section{Introduction}

Le taux d'inflation dans la zone euro a fortement baissé de $12 \%$ au début des années 1980 à $4 \%$ au début des années 1990. Malgré certaines différences, les taux d'inflation des pays de la zone présentent tous une tendance marquée à la baisse sur cette même période. Ces baisses des taux d'inflation ont fait suite soit à des politiques monétaires plus stables (par exemple en Allemagne), soit à des changements explicites dans la politique monétaire (par exemple, la politique de " désinflation compétitive " en France, voir Blanchard et Muet, 1993). Durant cette phase de désinflation, le taux de chômage a fortement augmenté et l'activité économique s'est notablement contractée dans la zone.

Certains auteurs supputent que les politiques de désinflation ont été à l'origine du ralentissement marqué de l'activité économique. Ainsi, pour Ball (1994), les périodes de désinflation sont l'une des causes majeures des grandes récessions aux États-Unis. En particulier, il est communément admis que la désinflation Volcker a été à l'origine de l'un des plus sévères ralentissements de l'activité aux États-Unis depuis la seconde guerre mondiale (Erceg et Levin, 2003). De façon plus générale, de nombreuses études empiriques mettent en évidence de fortes baisses du produit à la suite de politiques de désinflation (voir, entre autres, Ball, 1994, Cecchetti et Rich, 2001).

Cette question est reprise ici en partant du principe qu'une réponse convaincante nécessite la formulation et l'estimation d'un modèle économique de la zone euro au cours de cette période. Dans ce but, cette étude modélise la mise en œuvre complexe et hétérogène de la politique monétaire dans la zone sous la forme d'un processus stochastique non stationnaire dans lequel la cible implicite d'inflation (inobservée de l'économiste) de la banque centrale présente des changements graduels et permanents. La cible implicite d'inflation est alors assimilée aux mouvements de basse fréquence de l'inflation. L'hypothèse Friedmanienne sous jacente est que ces derniers relèvent intrinsèquement et exclusivement de la politique monétaire.

La cible implicite d'inflation est elle-même incluse dans une règle de Taylor généralisée. Cette règle stipule que la banque centrale augmente le taux d'intérêt nominal en réaction (i) à une modification de la cible implicite d'inflation, (ii) à des écarts de l'inflation à sa cible implicite, et (iii) à des écarts du niveau de la production à sa valeur potentielle.

Certes, pour l'essentiel de la période d'estimation considérée dans cette étude (1970(1)-2004(4)), les pays constituant la zone euro avaient chacun une banque centrale et ne mettaient pas en œuvre une politique monétaire unique. Le choix de modélisation retenu ici, d'ailleurs adopté dans la majeure partie de la littérature, est de traiter la banque centrale unique du modèle comme une banque centrale virtuelle, moyenne pondérée des 13 banques centrales nationales de l'époque. Cette approche a le défaut de gommer I'hétérogénéité des politiques monétaires précédant l'introduction de l'euro. Toutefois, cette approximation est cohérente avec la rapide adoption par de nombreux pays d'objectifs de politique monétaire convergents (en particulier, la réduction du niveau de l'inflation). 
La règle de politique monétaire boucle une petite maquette empirique de l'économie de la zone euro, inspirée des travaux de Rudebusch et Svensson (1999) et Gerlach et Smets (1999). Cette maquette comporte une équation d'offre (une courbe de Phillips) et une équation de demande (une courbe IS). La spécification des courbes IS et de Phillips impose la neutralité à long terme de la politique monétaire, et notamment de la cible implicite d'inflation. En dépit de sa simplicité, le modèle s'ajuste bien aux données et n'introduit que peu de restrictions théoriques.

A l'aide de données agrégées de la zone euro, relatives au taux d'intérêt nominal, à l'inflation et au PIB, sur la période 1970(1)-2004(4), l'estimation par une approche bayésienne de cette maquette macroéconométrique permet de répondre aux questions suivantes :

(i) Quelle a été l'évolution de cette cible implicite d'inflation au cours de la période d'estimation ?

(ii) Quels sont les effets sur le produit, l'inflation et le taux d'intérêt d'un choc négatif sur la cible implicite d'inflation?

(iii) Quelle est la contribution de ce choc aux fluctuations agrégées dans la zone euro ?

(iv) Quelle aurait été la dynamique des variables d'intérêt en l'absence de ces chocs ?

\section{Une maquette empirique de la zone euro}

\subsection{Présentation de la maquette}

Le modèle est synthétisé par 8 équations qui sont reportées dans l'encadré 1 et commentées ci-dessous. Les deux équations (1) et (2) déterminent la dynamique des composantes cycliques du produit, $\tilde{y}_{t}$, et de l'inflation, $\tilde{\pi}_{t}$. Ces deux composantes sont définies ici en écart à leurs tendances stochastiques respectives, ces dernières étant données par les équations (5) et (6).

L'équation (1) est inspirée de Rudebusch et Svensson (1999) et Gerlach et Smets (1999). Cette équation peut être vue comme une courbe IS qui relie l'écart de production à son propre retard et à la valeur retardée du taux d'intérêt réel ex post. L'équation est perturbée par un choc de demande $\varepsilon_{y, t}$, supposé indépendamment et identiquement distribué (i.i.d.), de moyenne nulle et de variance unitaire.

L'équation (2) est une courbe de Phillips qui relie la composante cyclique de l'inflation à son propre passé ainsi qu'à celui de l'écart de production. Cette équation est elle aussi perturbée par un choc stochastique, $\varepsilon_{\pi, t}$, qui s'interprète comme un choc d'offre transitoire. Ce choc est lui aussi supposé i.i.d., de moyenne nulle et de variance unitaire.

Le modèle est finalement bouclé par la spécification de la politique monétaire dans la zone euro, sous la 
forme d'une règle de Taylor généralisée ${ }^{3}$. Elle est détaillée dans les équations (3) à (5). L'équation (3) définit la cible de taux d'intérêt, notée $R_{t}^{*}$. Celle-ci est une fonction de la cible implicite d'inflation $\pi_{t}^{*}$, de l'écart de I'inflation courante à cette même cible $\tilde{\pi}_{t}$ et de l'écart du produit à son niveau tendanciel $\tilde{y}_{t}$. Les coefficients $a_{p}$ et $a_{y}$ mesurent la réactivité de la politique monétaire à ces deux dernières variables. L'équation (4) détermine l'ajustement partiel du taux d'intérêt nominal effectif de court terme $R_{t}$ à sa cible. Le paramètre $\rho$ détermine le degré de lissage de la politique monétaire. Cette équation d'ajustement est complétée par un terme aléatoire i.i.d. $\varepsilon_{R, t}$, de moyenne nulle et de variance unitaire. Finalement, l'équation (5) définit le processus suivi par la cible implicite d'inflation. Celle-ci est supposée non stationnaire, permettant de prendre en compte les mouvements de basses fréquences de l'inflation dans la zone euro ${ }^{4}$. La variation de la cible implicite d'inflation $\Delta \pi_{t}^{*}$ suit un processus autorégressif d'ordre un. Le coefficient autorégressif de la cible $\rho_{\pi^{*}}$ permet de prendre en compte l'ajustement graduel et persistant de la cible. Cette spécification de la politique monétaire est très proche de celle adoptée par Ireland (2007) dans le cadre d'un modèle plus structurel (DSGE).

L'équation (6) stipule l'évolution dynamique de la production potentielle. Le processus stochastique suivi par cette dernière capture les mouvements de basse fréquence du produit pouvant être engendrés par des chocs d'offre (chocs technologiques ou chocs permanents sur l'offre de travail, comme chez Chang, Doh et Schorfheide, 2007).

Au-delà de la spécification log-linéaire, la structure dynamique adoptée pour le modèle n'est pas arbitraire. En effet, des analyses préliminaires sur les équations (1) et (2) nous conduisent à sélectionner un nombre limité de retards dans ces dernières. Cette spécification parcimonieuse est suffisante pour capturer les grandes caractéristiques d'auto-corrélation des données. Le modèle étant purement tourné vers le passé, l'équation (2) implique que le taux d'inflation s'ajuste instantanément et intégralement au choc sur la cible d'inflation. Comme dans Ireland (2007), les entreprises réajustent le sentier des prix avec le changement de cible implicite. Une modification de cette cible est ainsi supposée connue instantanément par les agents privés. Cette hypothèse pouvant apparaître comme trop restrictive, nous avons alors amendé cette dernière en incluant le changement de cible $\Delta \pi_{t}^{*}$ dans la courbe de Phillips :

$$
\tilde{\pi}_{t}=\alpha \tilde{\pi}_{t-1}+\beta \tilde{y}_{t-1}+\kappa \Delta \pi_{t}^{*}+\sigma_{\pi} \varepsilon_{\pi, t} .
$$

Cette équation garantit que l'inflation et les autres variables nominales qui cointégrent avec elle ne

\footnotetext{
${ }^{3}$ On peut objecter à ce choix de modélisation que la plupart des pays qui constituent actuellement la zone euro n'ont sans doute pas recouru à une telle règle de politique monétaire durant la période couverte par notre échantillon. De facto, certains de ces pays affichaient des objectifs en termes de progression des encaisses monétaires ou en termes de taux de change. Sous certaines conditions théoriques, ces objectifs sont néanmoins équivalents à un ciblage du taux d'intérêt nominal. Le choix de modélisation qui est adopté dans cette étude reste donc valide et permet une paramétrisation parcimonieuse d'un processus en réalité très complexe.

${ }^{4}$ Les tests usuels de racine unitaire conduisent à ne pas rejeter l'hypothèse nulle de racine unitaire à des seuils conventionnels. La présence d'une racine unitaire s'avère une approximation statistique utile de la dynamique de l'inflation dans notre échantillon (1970(1)-2004(4)). Néanmoins, il apparaît moins raisonnable de maintenir cette représentation pour des échantillons plus longs ou en présence d'une politique monétaire plus stable, comme dans la période récente.
} 
s'écartent pas à long terme de leur tendance stochastique commune déterminée par l'évolution de la cible d'inflation. Le modèle est re-estimé avec cette nouvelle spécification de la courbe de Phillips et pour différentes distributions a priori du paramètre $\kappa$. Nous obtenons que ce paramètre n'est jamais significatif et que la vraisemblance marginale est quasiment identique à celle obtenue sur le système (1)-(8).

\section{Encadré 1}

\section{Les équations de la maquette}

(1) Courbe Is: $\tilde{y}_{t}=\varphi \tilde{y}_{t-1}-\lambda\left(R_{t-1}-\pi_{t-1}\right)+\sigma_{y} \varepsilon_{y, t}$

(2) Courbe de Phillips: $\tilde{\pi}_{t}=\alpha \tilde{\pi}_{t-1}+\beta \tilde{y}_{t-1}+\sigma_{\pi} \varepsilon_{\pi, t}$

(3) Règle de politique monétaire: $R_{t}=\rho R_{t-1}+(1-\rho) R_{t}^{*}+\sigma_{R} \varepsilon_{R, t}$

(4) Cible de taux d'intérêt : $R_{t}^{*}=\pi_{t}^{*}+a_{p} \tilde{\pi}_{t}+a_{y} \tilde{y}_{t}$

(5) Cible implicite d'inflation : $\Delta \pi_{t}^{*}=\rho_{\pi^{*}} \Delta \pi_{t-1}^{*}+\sigma_{\pi^{*}} \mathcal{E}_{\pi^{*}, t}$

(6) Production potentielle: $y_{t}^{p}=y_{t-1}^{p}+\sigma_{y^{p}} \varepsilon_{y^{p}, t}$

(7) Ecart de production: $\tilde{y}_{t}=y_{t}-y_{t}^{p}$

(8) Ecart d'inflation: $\tilde{\pi}_{t}=\pi_{t}-\pi_{t}^{*}$

$y_{t}$ est le produit, $R_{t}$ est le taux d'intérêt nominal, $\pi_{t}$ est le taux d'inflation. Les termes $\varepsilon_{y, t}, \varepsilon_{\pi, t}, \varepsilon_{R, t}, \varepsilon_{\pi^{*}, t}$ et $\varepsilon_{y}{ }_{y, t}$ sont des chocs aléatoires, supposés iid, de moyenne nulle et de variance unitaire.

Les signes attendus de certains des paramètres du modèle sont dictés par la théorie économique. En particulier, elle suggère les contraintes suivantes: $\lambda>0, \beta>0, a_{p}>1, a_{y}>0$. La première restriction théorique traduit la réponse négative de la demande agrégée au taux d'intérêt réel. La deuxième restriction est issue des relations de Phillips et d'Okun. La troisième restriction correspond au principe de Taylor qui stipule qu'une politique monétaire est stabilisante lorsque la cible de taux d'intérêt nominal répond plus que l'inflation aux différents chocs macro-économiques. La dernière restriction impose le caractère contracyclique de la politique monétaire.

Les paramètres $\sigma_{y}, \sigma_{\pi}, \sigma_{R}, \sigma_{\pi^{*}}$ et $\sigma_{y}$ sont des termes d'échelle permettant de mesurer la taille des différents chocs du modèle empirique.

\subsection{Discussion}

Le modèle présenté ci-dessus appelle certains commentaires. Tout d'abord, il spécifie un ensemble d'équations qui ne sont que des formes réduites de comportements structurels. Ainsi les anticipations des 
agents ne sont pas prises en compte et les paramètres structurels définissant les comportements des agents ne sont pas explicitement modélisés.

Suivant la critique de Lucas (1976), cela nous interdit de mener certaines analyses de politique économique, notamment des analyses contrefactuelles liées aux comportements des agents (par exemple, en modifiant un paramètre estimé). Cependant, l'analyse des réponses dynamiques de l'économie à des chocs nonanticipés reste valide, comme c'est le cas dans la méthodologie des modèles VAR structurel (Sims, 1980). Un avantage de notre approche en terme de représentation réduite est que l'identification de la cible implicite d'inflation est obtenue avec un nombre minimal de restrictions. Celles-ci sont simplement issues de la restriction théorique que l'inflation est uniquement un phénomène monétaire dans le long terme et que les chocs monétaires n'ont aucun effet réel à long terme. Il faut néanmoins mentionner que ces restrictions ne préjugent en rien des effets de court et moyen termes des chocs sur la cible implicite d'inflation.

Notons pour finir que le modèle n'impose aucune restriction sur le caractère crédible ou non de la politique de désinflation, au contraire d'un modèle plus structurel qui nous aurait contraints à nous prononcer sur cette question (voir Ireland, 2007, et Fève, Matheron et Sahuc, 2009). La plus ou moins grande crédibilité se retrouve en effet dans les paramètres de la forme réduite. Nous tirons ainsi un avantage de notre choix de spécification. Ceci est également vrai pour la méthodologie VAR structurel, mais celle-ci reste sujette à certaines critiques sur les schémas d'identification avec des restrictions de long terme (voir Dupaigne, Fève et Matheron, 2007, et Chari, Kehoe et McGrattan, 2007).

\section{Estimation et résultats}

\subsection{Approche économétrique}

Le modèle décrit précédemment est estimé par maximum de vraisemblance bayésien. Cette technique est récemment devenue un outil privilégié des macro-économistes (An et Schorfheide, 2007). L'idée générale de cette méthode est de combiner la vraisemblance du modèle économétrique avec des a priori sur les paramètres du modèle qui sont synthétisés sous la forme de distributions de probabilité. Cette procédure permet alors de calculer la distribution a posteriori des paramètres.

Les données utilisées dans l'analyse sont issues de la base AWM (Fagan, Henry et Mestre, 2005). La période d'estimation s'étend du premier trimestre 1970 au dernier trimestre 2004. Les variables observables retenues pour estimer le modèle sont (i) le taux de croissance du PIB par tête, (ii) la différence première du taux d'inflation, cette dernière étant définie comme le taux de croissance de l'indice harmonisé des prix à la consommation et (iii) le taux d'intérêt réel ex post, ce dernier étant simplement la différence entre le taux nominal de court terme et le taux d'inflation.

En pratique, nous adoptons les distributions a priori suivantes. Les paramètres $\alpha$, $\rho$, et le coefficient d'autocorrélation de la variation de la cible implicite d'inflation sont tous a priori distribués selon des lois Beta de 
moyenne 0,5 et d'écart-type 0,15 . Cette loi de distribution assure que ces paramètres sont compris entre 0 et 1 . Suivant la tradition en économétrie bayésienne, les écarts-types des chocs sont distribués selon des lois Gamma inverses, centrées sur 0,005 et d'écart-type 0,1. Ces valeurs assurent des a priori relativement diffus. Tous les autres paramètres sont $a$ priori distribués selon des lois normales. Les paramètres de ces lois sont choisis en cohérence avec les valeurs usuelles obtenues dans la littérature.

Des analyses complémentaires suggèrent que les résultats de l'estimation reportés ci-dessous sont robustes au choix des distributions a priori sur les paramètres du modèle.

\subsection{Résultats des estimations}

Les résultats de l'estimation sont reportés dans le tableau 1. Les valeurs reportées correspondent au mode de la distribution a posteriori et à l'écart-type de cette dernière.

Plusieurs résultats notables émergent de l'estimation. En premier lieu, la règle de Taylor est caractérisée par un fort degré de réponse à l'inflation (on parle dans la littérature de forte agressivité). Dans le même temps, le taux d'intérêt nominal répond de façon non négligeable à l'écart de production, traduisant une préoccupation de stabilisation de l'activité chez le banquier central. Enfin, la politique monétaire semble relativement inertielle, puisque le coefficient de lissage du taux d'intérêt est très élevé $(\rho=0,94)$. En revanche, I'ajustement dynamique de la cible implicite d'inflation semble plus rapide ( $\rho_{\pi^{*}}=0,34$ ).

\section{Tableau 1 Résultats de l'estimation du modèle}

\begin{tabular}{|l|l|c|c|}
\hline Paramètre & Interprétation & Mode & Ecart-type \\
\hline$\varphi$ & Persistance intrinsèque de l'écart de production & 0,9438 & 0,0223 \\
\hline$\lambda$ & Pente de la courbe IS & 0,1724 & 0,0432 \\
\hline$\alpha$ & Persistance intrinsèque de l'écart d'inflation & 0,1957 & 0,0799 \\
\hline$\beta$ & Pente de la courbe de Phillips & 0,1366 & 0,0319 \\
\hline$\rho$ & Degré de lissage du taux d'intérêt nominal & 0,9385 & 0,0144 \\
\hline$a_{p}$ & Élasticité du taux d'intérêt nominal ciblé à l'écart d'inflation & 1,6992 & 0,1486 \\
\hline$a_{y}$ & Élasticité du taux d'intérêt nominal ciblé à l'écart de production & 0,1403 & 0,0479 \\
\hline$\rho_{\pi^{*}}$ & Auto-corrélation des variations de la cible implicite d'inflation & 0,3430 & 0,1195 \\
\hline$\sigma_{\pi^{*}}$ & Écart-type des chocs de cible implicite d'inflation & 0,0011 & 0,0002 \\
\hline$\sigma_{R}$ & Écart-type des chocs monétaires & 0,0015 & 0,0001 \\
\hline$\sigma_{\pi}$ & Écart-type des chocs d'offre & 0,0023 & 0,0002 \\
\hline$\sigma_{y}$ & Écart-type des chocs de demande & 0,0052 & 0,0005 \\
\hline$\sigma_{y^{p}}$ & Écart-type des chocs sur le produit potentiel & 0,0024 & 0,0009 \\
\hline
\end{tabular}


Le coefficient $\alpha$, qui mesure la persistance intrinsèque de l'écart de l'inflation à sa cible implicite, admet une valeur relativement faible. La cible implicite d'inflation semble donc expliquer l'essentiel des propriétés de persistance à long terme de l'inflation agrégée de la zone euro.

Le coefficient $\beta$, qui mesure la pente de la courbe de Phillips, est faible $(\beta=0,14)$, suggérant un fort degré de rigidités nominales dans la zone euro. Ce résultat est assez similaire à celui obtenu par Gerlach et Smets (1999). Le coefficient $\varphi$, qui mesure la persistance intrinsèque de la demande, est relativement élevé $(\varphi=0,94)$, suggérant une forte inertie de la demande dans la zone. Dans le même temps, l'élasticité estimée de la demande au taux d'intérêt réel ex post $(\lambda=0,17)$ est plus forte que celle obtenue par Gerlach et Smets (1999).

\subsection{Analyse des effets agrégés des chocs de désinflation}

A partir de l'estimation des paramètres du modèle, nous menons différents exercices visant à étudier plus précisément l'évolution de la cible implicite d'inflation dans la zone euro et ses conséquences sur la dynamique agrégée.

\section{EVOLUTION DE L'INFLATION ET DE LA CIBLE IMPLICITE D'INFLATION}

Nous calculons dans un premier temps l'évolution de la cible implicite d'inflation issue du modèle estimé et nous la comparons à celle de l'inflation observée dans la zone euro. Le résultat de cette analyse est reporté dans le graphique 1. La cible implicite est obtenue en utilisant l'information contenue dans tout l'échantillon à l'aide du lisseur de Bucy-Kalman. Les séries chronologiques reportées dans ce graphique sont exprimées en taux annuels.

Comme le montre le graphique 1 , les deux séries ont des profils assez similaires, même si la cible ne rend pas compte de tous les mouvements de court et moyen termes du taux d'inflation. Ce résultat souligne simplement le fait que d'autres chocs (en particulier, des chocs d'offre défavorables) ont largement contribué à la dynamique de l'inflation dans la zone euro. En revanche, le comportement de basse fréquence de l'inflation est bien capturé par la cible. Celle-ci est en effet associée à la tendance haussière des années soixante-dix et à la forte baisse de l'inflation au cours des années quatre-vingts. 


\section{Graphique 1 Inflation annualisée et cible implicite}

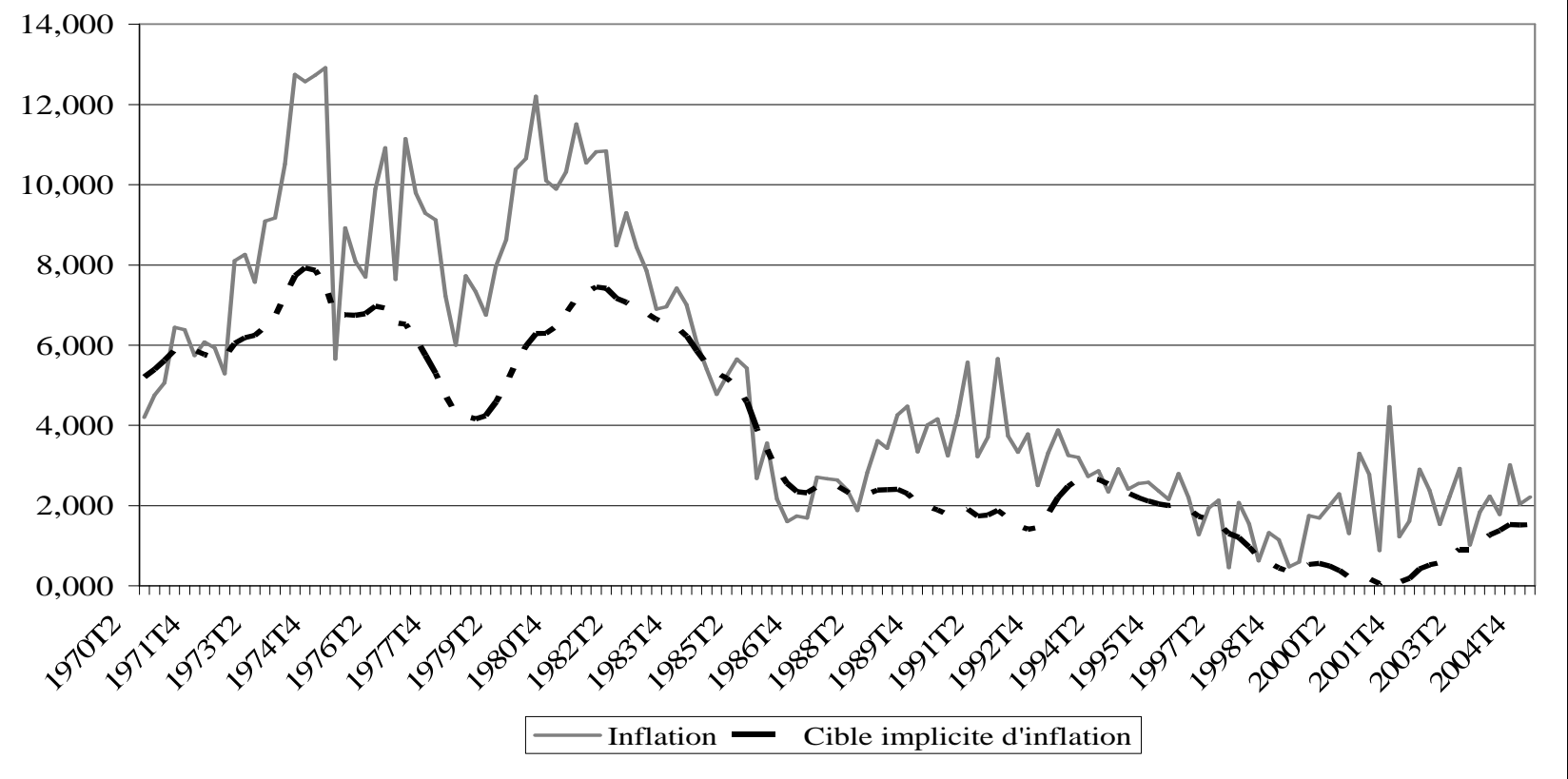

\section{FONCTIONS DE REPONSE A UN CHOC SUR LA CIBLE IMPLICITE}

Le graphique 2 reporte les fonctions de réponses dynamiques du produit, de l'inflation, du taux d'intérêt nominal et du taux d'intérêt réel (ex post). Ces réponses sont obtenues en appliquant un choc négatif sur l'innovation de la cible implicite d'inflation, ce qui revient à étudier la dynamique agrégée suite à une politique de désinflation.

L'inflation baisse régulièrement et présente un profil en cloche inversée. La réponse négative maximale est obtenue au bout de quatre ans. Plus de dix ans sont nécessaires pour que celle-ci se stabilise à sa nouvelle valeur de long terme. II convient de remarquer que la réponse dynamique de l'inflation présente plus de persistance que la cible implicite d'inflation elle-même. En effet, étant donnée la valeur estimée du coefficient d'auto-corrélation des variations de la cible implicite, cette dernière rejoint assez rapidement sa nouvelle valeur de régime permanent. Ces résultats suggèrent l'existence de mécanismes de propagation persistants des chocs de cible implicite d'inflation ${ }^{5}$.

\footnotetext{
${ }^{5}$ Comme souligné précédemment, les fonctions de réponse sont peu affectées lorsque nous incluons le changement de cible d'inflation contemporain dans la courbe de Phillips (équation 2).
} 


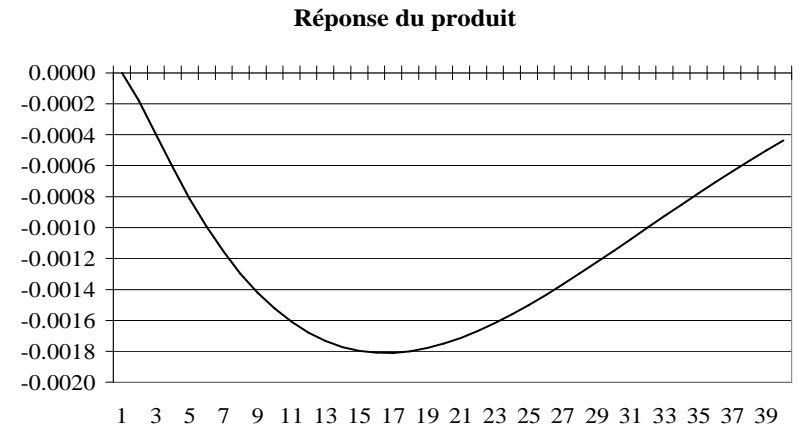

Réponse du taux d'intérêt nominal

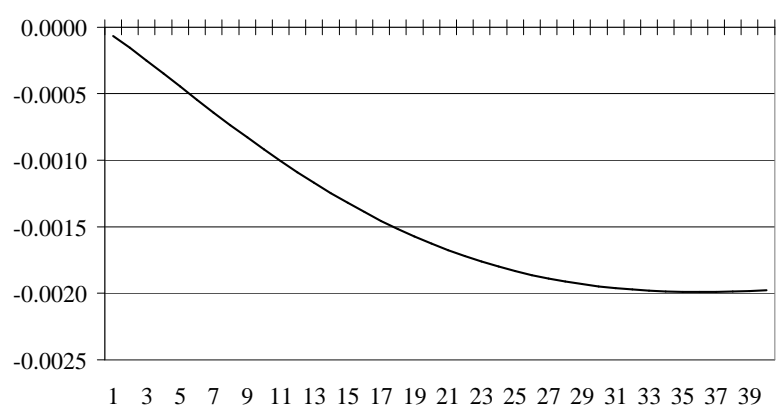

Réponse de l'inflation

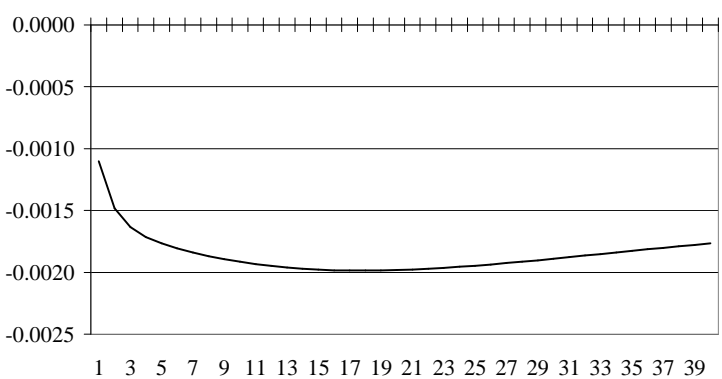

Réponse du taux d'intérêt réel ex post

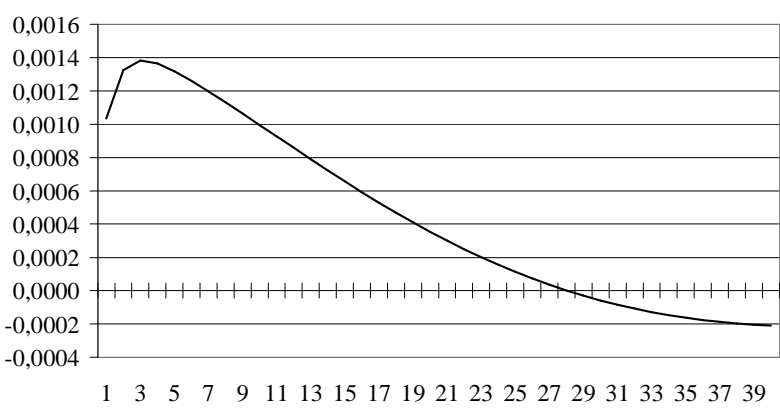

Dans le même temps, le taux d'intérêt nominal réagit très peu à court terme et baisse régulièrement vers sa nouvelle valeur de long terme. Le profil de cette réponse est essentiellement gouverné à court terme par le fort degré de lissage du taux d'intérêt nominal, qui en quelque sorte isole le taux des variations du taux cible. En conséquence, le taux d'intérêt réel ex post augmente instantanément et sa réponse positive est très persistante. II faut attendre environ sept ans pour que celui-ci baisse légèrement.

Comme attendu de nos estimations, le produit baisse de façon persistante suite au choc de désinflation. La réponse du produit présente un profil en cloche inversée. La réponse négative maximale est obtenue quatre ans après la survenance du choc.

Suite à un choc sur la cible d'inflation, la dynamique du produit est gouvernée par la hausse persistante du taux d'intérêt réel. A ceci s'ajoute la forte inertie estimée du comportement sur le marché des biens, telle que résumée par le paramètre $\varphi=0,94$. La dynamique inertielle du taux d'intérêt réel est ici une source essentielle des effets récessifs sur l'activité. Nos résultats soulignent ainsi les effets réels très persistants des chocs de désinflation, même si par construction ces chocs n'ont aucun effet à long terme sur le produit.

\section{CONTRIBUTION DU CHOC SUR LA CIBLE IMPLICITE}

Nous calculons ensuite la contribution de ce choc aux fluctuations agrégées dans la zone euro. Cet exercice est conduit pour différents horizons entre un et quarante trimestres. Les résultats sont reportés dans le 
tableau 2. Pour chaque variable et pour chaque horizon, le tableau donne la fraction de la variance totale expliquée par le choc sur la cible implicite d'inflation.

Au fur et à mesure que l'horizon augmente, le choc sur la cible implicite d'inflation représente (par construction) une part croissante de l'inflation et du taux d'intérêt nominal. Il faut cependant noter que ce choc explique à court terme l'essentiel des fluctuations du taux d'inflation, puisqu'il représente plus de $70 \%$ de la variance de l'inflation après deux ans. La contribution de ce choc à la dynamique du taux d'intérêt nominal est plus faible à court terme, illustrant ainsi la faible réaction de ce taux dans les périodes qui suivent le choc sur la cible implicite d'inflation. De façon remarquable, ce choc représente une part très importante des fluctuations du taux d'intérêt réel ex post. Par exemple, au bout de deux ans, sa contribution dépasse $40 \%$. Ce résultat est une conséquence directe de l'importante inertie du taux d'intérêt nominal de court terme couplée à l'ajustement relativement rapide de l'inflation à sa cible implicite. Concernant le produit, la part expliquée par le choc de désinflation reste plus modeste sans être négligeable (environ 10\%, après cinq ans).

Tableau 2 Décomposition de la variance

\begin{tabular}{|l|c|c|c|c|}
\hline \multicolumn{1}{|c|}{ Horizon } & Produit & Inflation & Taux d'intérêt nominal & Taux d'intérêt réel \\
\hline 1 & 0 & 18,876 & 0,195 & 14,163 \\
\hline 4 & 0,481 & 55,438 & 2,575 & 33,833 \\
\hline 8 & 2,433 & 70,108 & 10,778 & 40,556 \\
\hline 12 & 5,023 & 76,881 & 22,766 & 42,545 \\
\hline 20 & 9,534 & 84,042 & 45,850 & 41,610 \\
\hline 40 & 11,454 & 90,853 & 74,027 & 37,349 \\
\hline
\end{tabular}

\section{ANALYSE CONTREFACTUELLE}

Finalement, nous évaluons la contribution de la cible implicite d'inflation en menant l'exercice contrefactuel suivant. Nous cherchons en fait à répondre à la question suivante: "Quelles auraient été les évolutions dynamiques du produit, de l'inflation, du taux d'intérêt nominal et du taux d'intérêt réel, si la cible implicite d'inflation était restée constante durant toute la période d'estimation ? ".

Pour mener à bien cet exercice, nous imposons que l'innovation de la cible implicite soit nulle et nous recalculons les évolutions de ces quatre variables agrégées à l'aide du modèle macro-économétrique, aux valeurs estimées des paramètres. Les résultats sont reportés dans le graphique 3. 


\section{Graphique 3 Contrefactuels}
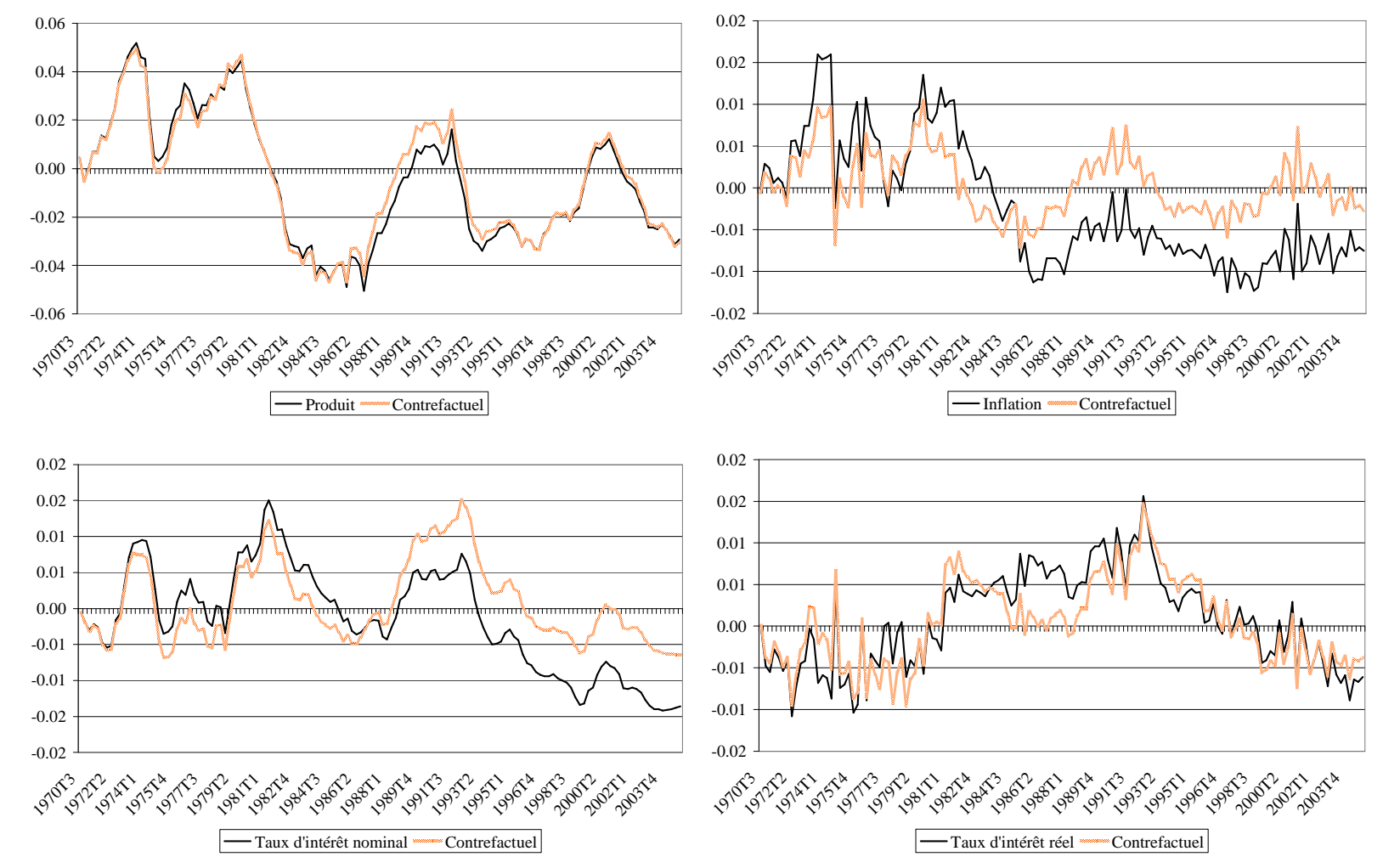

La graphique 3 présente l'évolution de l'inflation en présence et en absence du choc sur la cible implicite, après avoir centré les variables. Le graphique montre bien la contribution de ce choc. En effet, la nouvelle série d'inflation obtenue en mettant le choc à zéro s'écarte fortement de la série d'origine. En l'absence de ce choc, le modèle n'est plus à même de reproduire la tendance à la hausse des années soixante-dix. De façon plus remarquable, le modèle n'explique plus la forte baisse des années quatre-vingts. Ce graphique illustre le fait que les facteurs de basses fréquences (identifiés ici comme des chocs permanents sur la cible implicite d'inflation) ont fortement contribué à la dynamique de l'inflation dans la zone euro.

Le taux d'intérêt nominal présente un profil similaire à celui de l'inflation en l'absence du choc de désinflation, mais de façon moins marquée. Concernant le taux d'intérêt réel ex post, les années quatrevingts suggèrent que ce choc est un facteur important de leur hausse. Finalement, le choc de désinflation a un effet limité sur la composante cyclique du produit, puisque les deux séries diffèrent peu. ${ }^{6}$ ॥ faut cependant noter, que la zone euro aurait connu un niveau d'activité plus soutenu dans la seconde moitié des années quatre-vingts en l'absence du choc de désinflation, faisant ainsi écho à la hausse des taux d'intérêt réel durant cette période.

\footnotetext{
${ }^{6}$ Les résultats de nos estimations (non reportés ici) suggèrent que les chocs d'offre permanents sur le produit potentiel ont largement contribué aux fluctuations du produit à la fin des années soixante-dix et au début des années quatre-vingts.
} 


\section{Conclusion}

Dans cet article, nous avons proposé une maquette macro-économétrique dynamique de la zone euro incluant un choc inobservé, non stationnaire sur la cible implicite d'inflation. L'estimation de ce modèle suggère que ce choc a fortement contribué aux fluctuations des variables agrégées de la zone euro. Suite à un choc de désinflation, appréhendé ici comme une perturbation négative de l'innovation de la cible implicite d'inflation, le taux d'intérêt réel augmente de façon très persistante. Dans le même temps, le produit présente une baisse persistante, quoique d'amplitude modeste. De même, un exercice contrefactuel met en évidence le rôle joué par la cible implicite d'inflation, notamment au cours des années quatre-vingts.

\section{Bibliographie}

S. An et F. Schorfheide [2007] : Bayesian analysis of DSGE models, Econometric Reviews, pp. 26, 13-172.

L. Ball [1994] : What determines the sacrifice ratio?, In Mankiw (G.) éd., Monetary policy, pp. 155-88, The University of Chicago Press.

O. J. Blanchard et P-A. Muet [1993] : Competitiveness through disinflation: An assessment of French macroeconomic policy since 1983, Economic Policy, 16, pp. 12-56.

S. Cecchetti et R. Rich [2001] : Structural estimates of the U.S. sacrifice ratio, Journal of Business and Economic Statistics, 19, pp. 416-427.

Y. Chang, T. Doh et F. Schorfheide [2007] : Non-stationary hours in a DSGE model, Journal of Money, Credit, and Banking, 39, pp. 1357-1373.

V. V. Chari, P. Kehoe et E.R. McGrattan [2007] : Are structural VARs with long-run restrictions useful in developing business cycle theory?, Staff report 364, Federal Reserve Bank of Minneapolis

M. Dupaigne, P. Fève et J. Matheron [2007] : Avoiding pitfalls in using structural VARs to estimate economic models, Review of Economic Dynamics, 10, pp. 238-255.

C. Erceg et A. Levin [2003] : Imperfect credibility and inflation persistence, Journal of Monetary Economics, 50, pp. 915944.

G. Fagan, J. Henry et R. Mestre [2005] : An Area-Wide Model (AWM) for the euro area, Economic Modelling, 22, pp. 3959. 
P. Fève, J. Matheron et J-G. Sahuc [2009] : Inflation Target Shocks and Monetary Policy Inertia in the Euro Area, à paraître dans The Economic Journal.

S. Gerlach et F. Smets [1999] : Output gaps and monetary policy in the EMU area, European Economic Review, 43, pp. 801-812.

P. Ireland [2007] : Changes in the Federal Reserve's inflation target: Causes and consequences, Journal of Money, Credit, and Banking, 39, pp. 1851-1882.

R.E. Lucas [1976] : Econometric policy evaluation: A critique, Carnegie-Rochester Conference Series on Public Policy, 1, pp. $19-46$.

G.D. Rudebusch et L.EO. Svensson [1999] : Policy rules for inflation targeting, In Taylor (J.B.) éd., Monetary Policy Rules, pp. 203-246, The University of Chicago Press.

C.A. Sims [1980] : Macroeconomics and Reality, Econometrica, 48, pp. 1-48. 